\title{
Activités
}

14-1 | 2017

Varia

\section{Intervenir pour la conception de l'espace de l'activité : vers un cadre méthodologique}

Thinking and designing activity SPACE: towards a methodological framework

\section{Nadia Heddad}

\section{OpenEdition}

\section{Journals}

Édition électronique

URL : http://journals.openedition.org/activites/2958

DOI : 10.4000 /activites. 2958

ISSN : 1765-2723

Éditeur

ARPACT - Association Recherches et Pratiques sur les ACTivités

Référence électronique

Nadia Heddad, «Intervenir pour la conception de l'espace de l'activité : vers un cadre méthodologique », Activités [En ligne], 14-1 | 2017, mis en ligne le 15 avril 2017, consulté le 19 avril 2019. URL : http:// journals.openedition.org/activites/2958; DOI : 10.4000/activites.2958

Ce document a été généré automatiquement le 19 avril 2019

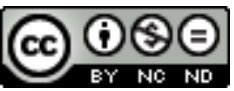

Activités est mis à disposition selon les termes de la licence Creative Commons Attribution - Pas d'Utilisation Commerciale - Pas de Modification 4.0 International. 


\title{
Intervenir pour la conception de l'espace de l'activité : vers un cadre méthodologique
}

Thinking and designing activity SPACE: towards a methodological framework

\author{
Nadia Heddad
}

\section{NOTE DE L'ÉDITEUR}

Article soumis le 6 février 2016, accepté le 18 novembre 2016

Remerciements à Marianne Cerf, Christine Chauvin et Pierre Falzon qui, de par leur exigence, m'ont aidée à préciser une pensée.

1 La visée de l'ergonomie est la transformation des situations de travail et le développement de l'activité. Cette ambition d'action a animé son orientation pour la conception et sa préoccupation pour le développement des méthodes pour l'intervention. Le modèle d'intervention en conception a été à l'origine développé en milieu industriel et a inspiré la conduite de projet industriel (Daniellou, 1988).

2 La conception des espaces de travail forme une modalité spécifique de l'intervention (Dejean, Pretto, \& Renouard,1988), mais intervenir sur l'espace peut se faire dans différents cadres. Le projet architectural est souvent affiché comme l'archétype de la conduite de projet en conception d'espaces. Il présente néanmoins une spécificité forte quant à la relation entre acteurs (propriétaires, concepteurs ou constructeurs de l'édifice) et s'inscrit dans un cadre projet spécifique ${ }^{1}$. Il est orienté tout entier pour faire aboutir, dans un temps donné, un objet commun, le bâtiment. Ainsi, Martin (2000) défend, dans le champ de l'ergonomie, une méthodologie spécifique en conduite de projet architectural. Il met la focale sur le travail de conception en architecture et propose un modèle pour l'intégration de l'ergonomie dans la conduite de projet architectural. La démarche d'intervention présentée par l'auteur est construite à partir d'un positionnement de 
l'ergonome dans le jeu d'acteurs particulier de la conduite du projet architectural ${ }^{2}$. La méthodologie proposée vise à cibler les occasions et les moments pertinents pour l'apport de l'ergonome à la conduite du projet.

D'autres manières d'aborder l'espace peuvent se présenter lors des interventions dans les organisations de travail sans nécessairement s'inscrire dans un projet de construction d'un bâtiment. Modifier une ligne de production, introduire de nouveaux outils technologiques dans le travail, réorganiser un service sont autant de situations qui modifient la dimension spatiale de la situation de travail.

Dans tous ces cas, l'intervention de l'ergonome touche l'espace, mais le concept d'espace est peu clarifié. L'espace est tantôt assimilé à l'architecture, au bâtiment, aux postes de travail, aux ambiances physiques des locaux et plus rarement à l'organisation. La dimension spatiale, reconnue en ergonomie comme partie intégrante de la situation de travail de l'activité, demeure peu conceptualisée (Heddad, 2016). Pour combler ce déficit, le concept d'espace de l'activité (Ibid.) propose une unité d'analyse pour saisir la dimension spatiale propre à chaque situation de travail. La visée est analytique et a pour ambition d'enrichir l'analyse de l'activité réalisée en ergonomie. Elle est aussi méthodologique en proposant un cadre pour l'intervention ergonomique permettant d'articuler les dimensions d'espace et d'activité pour la conception des situations de travail en considérant la spécificité de la dimension spatiale de l'activité. C'est le propos de cet article, qui après une définition de l'espace de l'activité, propose une démarche d'intervention pour la conception des situations de travail prenant en compte l'espace de l'activité.

5 Le texte est structuré en quatre parties. (i) Le concept espace de l'activité est présenté. Dans cette approche, l'analyse spatiale vient en complément à l'analyse de l'activité pratiquée en ergonomie. (ii) Un cadre méthodologique, organisé en deux temps, est proposé pour intervenir en conception. Des repères sont à construire au préalable à partir de l'analyse de l'activité combinée à l'analyse spatiale. La démarche est ensuite orientée de façon à viser le développement conjoint de l'activité et de l'espace de l'activité dans le travail de conception. (iii) Les limites et les apports de l'approche par l'espace de l'activité sont discutés. (iv) La perspective interroge l'apport d'une approche par l'activité dans la conception de façon générale.

\section{L'espace de l'activité}

\subsection{Définition}

$6 \quad$ L'espace de l'activité est à la fois un support et un produit de l'activité dont il ne peut être séparé. L'idée défendue est que toute activité se réalise dans un espace aux dimensions à la fois matérielles et relationnelles au travers des actions, des échanges entre les sujets ou entre les sujets et les objets qui les entourent lors de la réalisation de l'activité.

$7 \quad$ L'espace de l'activité est composé de deux registres : (i) une dimension matérielle relative à l'artefact, (ii) une dimension relationnelle émergente de l'activité.

(i) La dimension matérielle est artefactuelle et est tant physique qu'organisationnelle. Elle se décline en l'espace donné et l'espace modifié :

- L'espace est donné par l'organisation du travail. La dimension physique est reliée à l'organisation pensée et voulue par les concepteurs et l'organisation du travail. En effet, un 
espace se caractérise d'abord par sa matérialité, mais des choix d'organisation sont sousjacents à tout système spatial matériel. L'espace donné est alors organisateur de l'activité qu'il modifie et pour laquelle il est ressource ou contrainte.

- L'espace est modifié et ajusté lors de la réalisation de l'activité par les opérateurs qui recherchent à l'adapter à leurs contraintes. L'espace modifié et ajusté dans l'activité comporte d'une part, les réagencements physiques et organisationnels de l'espace matériel réalisés par les opérateurs et d'autre part, le développement des modalités et des règles de son utilisation.

9 (ii) La dimension relationnelle et immatérielle de l'espace de l'activité se compose de :

- l'espace mental de représentation de l'activité dans l'espace (l'approche est généralement au niveau individuel, mais peut aussi sur certains aspects concerner le collectif),

- l'espace émergent de l'activité au travers des relations, des coordinations, des coopérations et des formes de communication verbale ou non se créant dans le faire de l'activité (l'approche concerne tout aussi bien les modes d'échanges que les savoirs et les savoir-faire dans l'usage de l'espace de l'activité). Il s'agit d'une certaine manière de l'espace agi par l'activité.

10 L'espace de l'activité est modelé par l'activité dont il est à la fois le support et le produit. Il contribue au processus de réalisation de l'activité dont il fait partie.

\subsection{Analyse spatiale et espace de l'activité}

11 Pour traiter de l'espace de l'activité en conception, la dimension spatiale de l'activité est à aborder dès la phase d'analyse. Réaliser une analyse spatiale est incontournable si la visée consiste à contribuer à la conception d'un espace pensé pour accueillir une activité. L'analyse spatiale met la focale sur la dimension spatiale propre à chaque situation de travail, sa spécificité et sa relation à l'activité. Elle permet d'instruire et de rendre compte de la dimension spatiale de la situation de travail. En étudiant et explorant la relation entre la réalisation de l'activité et l'espace considéré comme ressource ou contrainte dans la réalisation du travail, elle apporte un éclairage sur l'articulation entre l'espace et l'activité en situation. Elle vient ainsi enrichir l'analyse du travail réalisée en ergonomie.

Cette mise en relation entre d'un côté, le déroulé de l'activité et de l'autre, la mise en œuvre de l'activité dans l'espace est rarement explicitée par les opérateurs. Elle peut cependant faire l'objet d'observations pour peu que l'outillage analytique s'y intéresse et intègre une approche par l'espace de l'activité. Comme le souligne Lautier, «l'étude de l'espace permet d'atteindre des aspects qui demeurent ordinairement dans l'implicite, le non-dit » (2001, p. 109).

Pour ce faire, l'analyse est à structurer à partir des deux registres précédemment évoqués.

La dimension matérielle de l'espace physique et organisationnel peut être abordée au travers d'une double lecture :

- L'espace donné par l'organisation du travail. Cet espace est « donné » au sens de Lave (1988). Il s'agit de l'espace conçu (Lefebvre, 1974) par les décideurs et les concepteurs. C'est de ce point de vue un artefact pensé par l'organisation et/ou les concepteurs. L'artefact est physique (éléments du bâti, mobilier, équipements, signalétique, etc.), mais il est aussi organisationnel (Arnoud \& Falzon, 2013).

- L'espace modifié et ajusté par l'activité. Il s'agit de l'espace transformé par les opérateurs qui adaptent l'espace physique et organisationnel mis à leur disposition. L'espace peut ici 


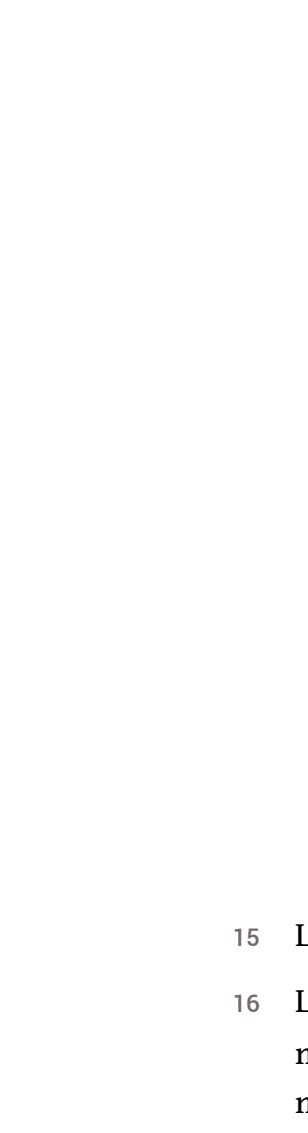
mentale de l'espace et de l'activité. Il s'agit alors d'un espace mental, celui que se crée
mentalement un opérateur par exemple lors de la conduite de systèmes complexes
(Amalberti, 1996). L'approche concerne les processus de résolution de problème
rencontré lors de la conduite des systèmes. Elle ne fait pas nécessairement le lien avec
l'espace matériel et ne sera pas exploitée dans nos propos. mentale de l'espace et de l'activité. Il s'agit alors d'un espace mental, celui que se crée
mentalement un opérateur par exemple lors de la conduite de systèmes complexes
(Amalberti, 1996). L'approche concerne les processus de résolution de problème
rencontré lors de la conduite des systèmes. Elle ne fait pas nécessairement le lien avec
l'espace matériel et ne sera pas exploitée dans nos propos. mentale de l'espace et de l'activité. Il s'agit alors d'un espace mental, celui que se crée
mentalement un opérateur par exemple lors de la conduite de systèmes complexes
(Amalberti, 1996). L'approche concerne les processus de résolution de problème
rencontré lors de la conduite des systèmes. Elle ne fait pas nécessairement le lien avec
l'espace matériel et ne sera pas exploitée dans nos propos.

Le deuxième niveau, représenté par le modèle de l'activité médiatisée par les instruments de Rabadel (1995), fait le lien entre l'activité individuelle et collective au travers des trois formes d'orientation de la médiation. (i) Les médiations vers l'objet permettent au sujet de comprendre et connaitre l'objet de son activité dans une visée épistémique, mais aussi d'agir et de transformer cet objet dans une visée pragmatique. (ii) Les médiations entretenues avec le sujet lui-même dans une boucle réflexive. (iii) Les médiations orientées vers les autres sujets dans une approche collective de l'activité. Appliquée à l'espace cette dernière déclinaison des intermédiations permet de distinguer l'activité réalisée individuellement, mais qui médiatise une relation adressée à un collectif. Il s'agit de toutes les traces spatiales de l'activité qui permettent une intelligibilité de l'activité réalisée par chacun. L'objectif est la coordination des activités de chacun par le recours à la manière d'agencer l'activité et ses traces dans l'espace. Ces traces spatiales peuvent aussi servir à ancrer l'activité d'autres sujets sans qu'ils ne se rencontrent. L'objectif est alors de coordonner une activité collective de coopération à partir des traces spatiales de l'activité. Un lien avec la notion de situation awarness ${ }^{4}$ (conscience de la situation) développée par Endsley (1995) et ayant fortement inspiré les études dans l'aéronautique dans l'étude des processus cognitifs en environnement dynamique (Chalandon, 2007; Salembier \& Zouinar, 2004) peut ici être opéré. En effet, le rapport entre l'espace physique et organisationnel et la représentation mentale des individus d'un collectif peut s'étudier sous l'angle de l'espace utilisé par les individus pour une mise en visibilité pour le collectif de ce qu'est la situation. Ce faisant, la manière de recourir à l'espace agencé d'une certaine manière ou de se placer dans l'espace (dans une certaine posture) permet « l'accès des agents à l'activité des autres agents dans la gestion collective des activités » (Salembier \& Zouinar, 2004).

Le dernier est une approche collective de l'espace de l'activité autour des relations, des interactions et des formes de communication verbale ou non qui se créant dans le faire de

\begin{abstract}
être analysé sous l'angle de sa qualité à constituer un appui, ou à l'inverse, une difficulté pour l'activité. Dans la réalisation de l'activité, l'espace physique et organisationnel est une contrainte ou une ressource (Lautier, 1999). L'espace donné, en présentant des qualités, offre des affordances au sens de Norman (1993) pour des usages possibles. Il peut prendre le statut d'un instrument lorsqu'un schème ${ }^{3}$ d'utilisation lui est associé (Béguin, 2006 ; Bourmaud, 2013 ; Rabardel, 1995 ; Vergnaud, 1996). Dans cette approche, l'instrument est l'artefact composé de l'espace physique et organisationnel modifié par les opérateurs pour répondre aux besoins de leur activité, auquel est associé un schème d'utilisation. L'idée est ici de s'intéresser au processus d'appropriation des artefacts dans une visée instrumentale (Folcher \& Rabardel, 2004) appliquée ici aux éléments constitutifs de l'espace matériel. Les composantes du bâti (poteaux, cloisons, portes, ascenseurs, etc.) peuvent être utilisées comme appui à l'action lors de la réalisation de l'activité. Les opérateurs peuvent aussi opérer des modifications dans l'affectation des fonctions des locaux opérant ainsi des genèses instrumentales (Clot \& Gori, 2003 ; Lefort, 1982 ; Rabardel, 1995).
\end{abstract} interions des forses decon 
l'activité. Il s'agit d'un espace émergeant dans l'activité. Il est de ce point de vue, une production locale au sens de Suchman (1996). Dans son étude shared workspaces, l'auteur montre comment l'espace se construit et émerge de l'activité et forme à la fois un tout commun et des espaces différenciés pour l'activité : « L'espace de travail offert par la salle des opérations n'est pas seulement donné par la disposition intérieure de la pièce, mais est le résultat d'une collaboration impliquant la production et la transformation continue de l'espace personnel, des espaces occupés en commun et des frontières entre eux. En tant que centre de coordination d'activités distribuées dans l'espace et le temps, la salle des opérations manifeste, dans son agencement, les exigences opposées du travail en commun et de la division du travail, celle aussi d'un foyer unique et commun et d'un ordonnancement discontinu d'espaces de travail différenciés " (Suchman 1996, p. 57, traduite par Quéré, 1997, p. 178). L'analyse est à orienter sur les modes d'interaction au sens de Goffman (1974). L'approche interactionniste ${ }^{5}$, permet de mettre la focale sur la manière dont l'action se construit entre les personnes en relation avec le lieu dans lequel elle opère. Les gestes, les mimiques, les façons de se mouvoir dans l'espace lors du faire dans la réalisation de l'activité sont à prendre en compte. L'analyse est à compléter par les modes de coordination et de communication au sens de la proxémie $^{6}$ de Hall (1966/1971). L'approche proxémique met en lumière les relations implicites composées des interdistances entre personne ou entre personnes et objets lors de la réalisation de l'activité. La distance entre opérateurs qui coopèrent dans l'activité n'est pas la même que pour des équipes mises en concurrence. De la même façon, la distance du contremaitre ou des hiérarchiques par rapport aux opérateurs est porteuse de sens intelligible par les acteurs concernés. Des savoirs et des savoir-faire sont sous-jacents à ces deux modes de relation et d'échange (interactions et proxémie). Ces aspects ne sont pas explicites dans une organisation, mais ils peuvent faire l'objet d'observations. Les communications non verbales guident et influencent l'action dans une situation locale (Varela, Thompson, \& Rosch, 1993). Ces relations sont à prendre en compte dans leur effet de structure par rapport à la situation de travail reliant l'activité et l'espace, autrement dit, en considérant l'espace de l'activité.

19 Pour conclure ici, l'analyse spatiale instruit la dimension spatiale de l'activité et apporte un éclairage qui vient enrichir l'analyse de l'activité pratiquée en ergonomie.

\section{Cadre méthodologique pour Intervenir en conception sur l'espace de l'activité}

Cela a été évoqué, le concept d'espace de l'activité invite à une conception conjointe de l'activité et de son espace dans un processus de transformation réciproque. L'objet de la conception est orienté à partir d'un couplage entre dynamique de l'activité (construite à partir d'exigences propres à la production et aux individus) et dynamique spatiale de l'activité.

21 À partir de la définition de l'espace de l'activité, nous proposons un cadre pour une démarche en conception. Elle est structurée en deux temps : (i) concevoir l'espace comme un dispositif physique et organisationnel adapté à l'activité, (ii) construire et mettre en œuvre un dispositif d'intervention visant le développement conjoint de l'activité et de l'espace de l'activité. 
La démarche est illustrée au travers d'exemples (en encadré ou sous forme de tableaux). Ils sont issus d'une intervention recherche réalisée en milieu postal ayant fait l'objet d'un article précédent (Heddad, soumis). Même si l'espace et l'activité ont été fortement modifiés par les choix de l'entreprise en matière d'organisation et de modèle spatial, les deux préexistent et servent de repères dans le cas de la démarche proposée en conception.

L'entreprise est une grande entreprise française de traitement du courrier. La demande est centrée sur l'espace suite à la modification du modèle d'organisation et des espaces. Elle vise à comprendre les plaintes exprimées par les équipes dans les nouveaux centres de traitement du courrier. Le périmètre d'analyse a concerné tout aussi bien des centres présentant des difficultés de performance (qualité et productivité) et des centres, anciens ou récents, considérés par la structure comme performants. Au total, six centres ont été étudiés et trois ont fait l'objet d'observations détaillées portées sur la relation entre l'espace et l'activité. L'analyse a ciblé tant des centres ayant modifié le bâti et l'organisation spatiale que des sites ayant adopté un nouveau modèle spatial autour du hall industriel.

\subsection{Repères pour concevoir un espace matériel adapté à l'activité}

L'objectif est ici d'engager un travail d'élaboration de critères pour guider les choix de conception de l'espace considéré comme un artefact. Il s'adresse à des concepteurs et des décideurs.

La conception de l'espace matériel, considéré comme un dispositif physique et organisationnel, peut se décliner en trois étapes :

- Définir les invariants organisationnels qui structurent l'activité de travail dans le temps et l'espace quel que soit le site.

- Repérer les objectifs de travail et les invariants opératoires reliés aux schèmes d'utilisation de l'espace physique et organisationnel.

- Traduire les objectifs et les invariants opératoires en objectifs à satisfaire par la conception.

Les éléments issus de la réflexion peuvent être intégrés à l'élaboration d'un cahier des charges en vue de la conception de l'espace de l'activité considéré comme un dispositif matériel. Il s'agit d'une première transposition de l'analyse du travail enrichie par l'analyse spatiale en vue d'une démarche de conception.

\subsubsection{Définir les invariants organisationnels de l'activité}

L'activité de travail est structurée par des invariants organisationnels qui la déterminent quel que soit le site. Ils orientent et structurent l'activité de l'opérateur en situation de travail. Pour les distinguer des déterminants de l'activité, nous les avons qualifiés d' invariants organisationnels, car ils sont à prendre en compte quel que soit le bâti et indépendamment de la typologie de l'organisation des espaces. Ils sont propres à la logique de l'activité et constituent une catégorie particulière des déterminants de l'activité. Ils guident l'action des opérateurs. Ils sont des composants du réel intégrés par les agents et à partir desquels ils structurent leur manière de faire face aux contingences de la situation et cela dans une perspective d'efficacité au travail. 
L'étude du travail de tri postal évoquée précédemment montre que l'activité est issue d'une triple préoccupation se déclinant de façon singulière dans chaque situation locale de chacun des centres de traitement du courrier. L'action des équipes est structurée de façon à prendre en compte 3 invariants organisationnels indépendamment du bâti :

- Les exigences de conditionnement en lien avec les choix d'acheminement et de transport pour connecter un réseau local à un réseau national ou international. La conteneurisation dépend du mode d'acheminement (voie routière, ferrée ou aviation). En fonction, les opérateurs manipulent des conteneurs paquets, des structures à bacs à lettres ou des sacs.

- La structure temporelle. En fonction des différents horaires prévus pour les arrivées et les départs des produits du centre, mais aussi en intégrant les aléas temporels liés au transport (intempérie ou retard de liaison par exemple), les agents disposent d'une plage horaire pour réaliser leur activité. Elle conditionne leur manière d'organiser les priorités des lots. Ils visent la prise en charge de l'ensemble des lots, mais doivent toutefois opérer des choix selon les temps impartis et hiérarchiser les priorités en fonction des urgences et des quantités à trier. Précisons que les agents n'ont pas de visibilité sur les volumes qu'ils doivent traiter. Ils découvrent au fur et à mesure de l'arrivée des camions les volumes des flux à prendre en charge.

- Les exigences techniques relatives au fonctionnement des machines de tri. Le mode de préparation et de présentation des produits est propre à chaque type de machine. Les agents ont la mission d'homogénéiser la présentation des plis et de lots de façon à satisfaire les exigences techniques de chaque machine et permettre ainsi un traitement optimisé en évitant les incidents techniques.

Ces invariants organisationnels ${ }^{7}$ forment un cadre qui oriente l'action des opérateurs dans la façon qu'ils ont d'avoir recours à l'espace matériel. Ils orientent les utilisations possibles de cet espace physique, organisé et organisateur de leur activité. C'est cela que nous tenterons de mettre en lumière dans ce qui suit.

\subsubsection{Repérer les objectifs de travail et les invariants opératoires reliés aux schèmes d'utilisation de l'espace physique et organisationnel}

Lors de la réalisation de leur activité, les opérateurs ont recours à l'espace (physique et organisationnel) d'une façon précise. Ils élaborent des schèmes d'utilisation (Rabardel, 1995, Vergnaud, 1996) de cet espace physique et organisationnel dans l'action.

En référence à la définition de Vergnaud $(1996,2013)$ le schème peut être décliné en quatre composantes : l'intention (buts, sous buts et anticipations), les règles (d'action, de 
prise d'information et de contrôle), les invariants opératoires et les possibilités d'inférences en situation.

Rapportée à l'espace matériel, cette définition du schème est revisitée en conception pour penser les gestes et les raisonnements des opérateurs en situation de travail en lien avec l'espace. Elle se décline alors de la façon suivante :

- Les invariants organisationnels, déclinés localement, sont traduits par les équipes en buts. Ce sont des objectifs de travail que les opérateurs s'assignent. Ils orientent l'action collective. Ils sont partagés dans l'activité de coopération entre opérateurs. Ces objectifs ou buts issus et déclinés des invariants organisationnels motivent l'action locale des équipes qui agissent en ayant recours aux moyens dont ils disposent. L'espace physique et organisationnel fait partie intégrante de ces moyens. Ces buts sont spatialement matérialisés.

- Les règles d'action (ou de prise d'information ou de contrôle) partagées par les équipes, quel que soit le site, concernent les règles de priorité à respecter et à mettre en œuvre dans le travail en fonction des aléas et des exigences temporelles de l'activité.

- Les buts adossés aux règles guident la structuration et la mise en œuvre de l'activité qui s'opère sur la base d'invariants opératoires. Ces invariants opératoires, souvent mal (ou peu) explicités par les équipes, sont observables dans la manière d'occuper et d'utiliser l'espace physique et organisationnel donné ou conçu par l'organisation et les concepteurs. Nous proposons de les qualifier d'invariants opératoires spatiaux.

- Les règles d'ajustements guidant les opérateurs dans la traduction des buts, des règles ou des invariants opératoires pour faire face à l'aléa en situation réelle peuvent être considérées comme les « possibilités d'inférences » de Vergnaud (1996, 2013).

En conception, la démarche proposée ici restreint l'étude aux composantes du schème que sont les buts et les invariants. Ils sont tous deux en lien avec l'artefact spatial. Les règles d'action ou d'inférences sont relatives à la dimension temporelle de l'activité. Elles sont sous-jacentes à l'action qui prend appui sur l'artefact spatial, mais elles ne le modifient pas.

Pour illustrer, des exemples issus de l'analyse réalisée dans le tri postal montrent quelques schèmes d'utilisation de l'espace structurant l'action collective et individuelle des équipes.

- L'activité des agents au niveau du quai arrivée est un travail de tri et d'aiguillage des flux qui suppose de repérer, filtrer, contrôler et hiérarchiser les lots de courrier selon les différentes priorités pour ensuite les orienter en vue de leur prise en charge sur les différentes machines de tri. Les portes et la cloison séparant l'intérieur de l'extérieur du quai sont utilisées comme support au travail de filtrage et de hiérarchisation des flux. Par l'utilisation du bâti au travers du jeu d'utilisation des portes et l'affectation des zones de part et d'autre de la cloison séparant l'intérieur de l'extérieur du quai, les agents affectent une fonction à l'espace physique. Le bâti est utilisé comme appui. Les agents en font usage d'une certaine façon dans la structuration de leur activité. Il s'agit d'un schème d'utilisation du bâtiment répondant au besoin de filtrage, de contrôle et de hiérarchisation des lots. Filtrer, contrôler et hiérarchiser en continu les lots au fur et à mesure de leur déchargement par les véhicules arrivant au centre est un objectif de travail auquel le bâti et les agencements intérieurs doivent satisfaire. Dans une approche instrumentale, les éléments du bâti (quai, portes, cloisonnements et ascenseurs) 
contribuent à l'activité instrumentée de filtrage et de hiérarchisation des lots en fonction des priorités qui s'imposent aux agents.

- L'analyse montre également que l'une des activités stratégiques en termes de performance satisfaisant les exigences de qualité (traiter les produits selon leurs priorités dans les temps) consiste à gérer la localisation spatiale dans le temps des différents stocks. Pour ce faire, les agents organisent visuellement la progression de la prise en charge des lots. Cela se décline en une manière d'organiser les lots dans les locaux selon leur état de prise en charge : les lots urgents, les encours ou les stocks en attente sont appuyés, adossés ou placés d'une certaine façon par rapport aux éléments du bâtiment que ce soit les murs, les poteaux ou les ascenseurs. Le schème consiste à utiliser les différentes zones du bâtiment en fonction de leur localisation et des caractéristiques des éléments d'architecture le composant (poteaux, mur, ascenseur, quai, etc.). Le bâti offre ici des qualités permettant la mise en place d'un parcours spatial des lots à placer de façon distincte et clairement identifiable par les équipes. Organiser ce parcours en zones différenciées pour accueillir des lots à état variable dans la prise en charge est une réponse construite dans l'action des agents. Dit autrement et dans une approche instrumentale, les agents construisent un schème d'usage dans la manière de différencier les zones des stockages et des encours des lots agencés d'une certaine façon dans l'édifice et cela pour répondre aux exigences temporelles qui s'imposent à eux. Les lots sont spatialement localisés et agencés d'une manière précise dans des zones clairement identifiées par eux (le long d'une cloison, autour d'un poteau ou regroupés selon une configuration géométrique spécifique). Ce faisant, c'est en s'appuyant sur l'espace matériel qu'ils construisent des règles d'action pour hiérarchiser les priorités dans le temps contraint de la production.

- À la fermeture des machines de tri, les agents doivent acheminer sans erreur les produits traités vers les véhicules prévus pour le départ des flux du centre. Pour cela, il leur faut faire converger les flux des produits traités en fin de production des machines. Ces dernières sont disposées de façon éclatée dans le centre. Cette exigence de travail se décline par le schème consistant à rassembler les produits dans une même zone en vue de leur organisation par destination. Le schème d'utilisation consiste à recourir à un goulet d'étranglement dans l'acheminement des flux. Dans les sites anciens sur deux niveaux, l'ascenseur est utilisé comme moyen de convergence. Les équipes rassemblent tous les flux face à l'ascenseur et les font descendre par ordre de priorité. Pour contrôler, organiser et hiérarchiser les flux par destination selon les camions en partance, ils ont à nouveau recours au système de portes donnant sur le quai combiné à la cloison séparant l'intérieur de l'extérieur du quai, mais dans le sens inverse par rapport à l'arrivée pour organiser cette fois les flux en partance par destination.

- La modification ponctuelle de l'affectation du quai des arrivées en quai pour gérer des départs dans la nuit est une catachrèse (Lefort, 1982 ; Rabardel, 1995 ; Clot \& Gori, 2003) modifiant provisoirement la fonction du quai. Ce détournement de fonction est une genèse instrumentale correspondant à une tactique opératoire des agents pour faire face et gérer un volume important de produits traités qu'il leur faut organiser par destination dans un temps extrêmement contraint (20mn). En modifiant l'affectation du quai prévu pour les arrivées en quai de départ, ils 
parviennent à gérer la contrainte temporelle pour un dégagement massif, mais contrôlé des flux qu'ils aiguillent par destination de véhicule tout en ménageant la possibilité de continuer à préparer, sur le quai officiel des départs, le reste des produits à faire partir dans une temporalité détendue. Ils disposent alors de plus d'une heure et demie pour cette préparation des départs de plis triés sur le quai de départ et non plus de $20 \mathrm{mn}$ comme évoqué précédemment. En fin de nuit, les agents utilisent les deux quais pour les départs. L'un est celui prévu officiellement pour les départs. L'autre, le quai des arrivées fait l'objet d'une utilisation informelle au sens de Lefort (1982) dans une optique d'efficacité au travail le temps de faire partir les flux les plus contraignants en termes d'exigence temporelle. Les agents en modifiant provisoirement l'affectation du quai arrivée en quai départ, dédoublent la ressource spatiale en quai. Le quai prévu pour les arrivées est utilisé pour gérer les départs de la première vague de courrier traités dans un temps particulièrement contraint tout en préservant le quai des départs pour la préparation de la seconde vague de courrier triés.

Pour résumer, la déclinaison de la définition du schème appliquée à l'espace conduit à décliner le raisonnement représenté dans la figure 1: À partir de l'activité, il s'agit de repérer les liens entre les buts, les invariants opératoires et les artefacts spatiaux constitués par les éléments de l'espace physique et organisationne.

Figure 1 : Identification des buts, des invariants opératoires et des artefacts spatiaux utilisés (processus de raisonnement).

Figure 1: Identification of goals, operational invariants and the space elements used

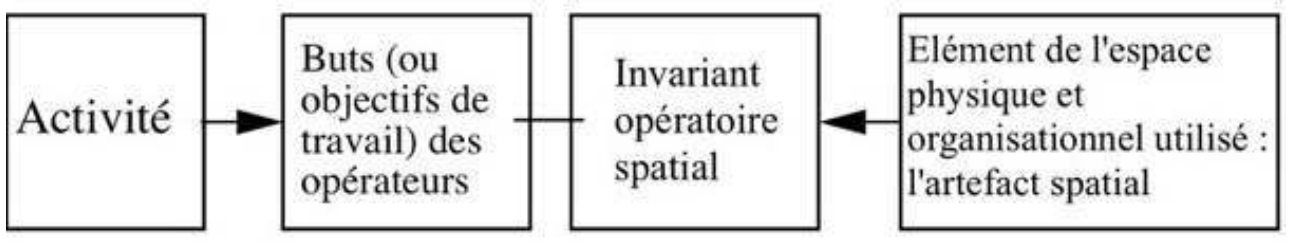

À partir de la compréhension de l'activité, des artefacts spatiaux peuvent être identifiés. Des invariants opératoires spatiaux peuvent alors en être déduits. Le principe de cette déclinaison permet de renseigner un tableau des artefacts spatiaux et des invariants opératoires associés. L'objectif est de repérer les éléments de l'espace physique et organisationnel assurant un rôle d'appui à l'activité. L'enjeu est une mise en lisibilité des relations qui relient l'activité et l'espace modifié et ajusté par les opérateurs en situation de travail.

L'exemple de l'activité de tri postal est utilisé à nouveau pour illustrer une manière de renseigner le Tableau 1. Il synthétise le lien entre les buts, les invariants opératoires et les éléments de l'espace physique et organisationnel et montre les éléments de l'espace assurant un rôle d'appui à l'activité dans le cas du travail de tri ${ }^{8}$. 
Tableau 1 : Déclinaison des buts, des invariants opératoires et des éléments spatiaux utilisés dans le cas de l'activité de tri postal.

Table 1: List of goals, operational invariants and space elements used in the case of the mail sorting activity

\begin{tabular}{|c|c|c|c|}
\hline Activité & $\begin{array}{l}\text { Buts ou objectifs de } \\
\text { travail des opérateurs }\end{array}$ & Invariants opératoires spatiaux & $\begin{array}{l}\text { Élément de l'espace } \\
\text { physique r et } \\
\text { organisationnel } \\
\text { utilisé : artefact } \\
\text { spatial }\end{array}$ \\
\hline $\begin{array}{l}\text { Tri et } \\
\text { aiguillage des } \\
\text { flux. }\end{array}$ & $\begin{array}{l}\text { Repérer, filtrer, } \\
\text { contróler et } \\
\text { hiérarchiser les lots de } \\
\text { courrier selon les } \\
\text { différentes priorités. }\end{array}$ & $\begin{array}{l}\text { Recours à la cloison séparant } \\
\text { l'intérieur de l'extérieur du quai et d'un } \\
\text { système de jeu de portes comme moyen } \\
\text { de séparation des produits : } \\
\text { - Sur le quai : les lots en attente d'une } \\
\text { prise en charge. } \\
\text { - De l'autre cóté de la cloison : les lots } \\
\text { sont pris en charge un à un par les } \\
\text { agents. } \\
\text { - Recours à une seule porte: toujours } \\
\text { celle placée face à la zone de travail } \\
\text { des agents. }\end{array}$ & $\begin{array}{l}\text { Le quai des arrivées, la } \\
\text { cloison séparant } \\
\text { l'intérieur de } \\
\text { l'extérieur du quai et le } \\
\text { système de jeu de } \\
\text { portes. }\end{array}$ \\
\hline $\begin{array}{l}\text { Gestion des } \\
\text { stocks selon les } \\
\text { priorités. }\end{array}$ & $\begin{array}{l}\text { Organiser visuellement } \\
\text { la progression de la } \\
\text { prise en charge des lots } \\
\text { et faire converger les } \\
\text { fivx des produits traités } \\
\text { en fin de production. }\end{array}$ & $\begin{array}{l}\text { Mise en place d'un parcours de zones } \\
\text { identifiées pour chaque niveau } \\
\text { d'urgence ou de prise en charge. } \\
\text { Délimitation de zones pour les lots } \\
\text { urgents, les encours ou les stocks en } \\
\text { attente en utilisant les éléments du bati } \\
\text { (murs, poteaux ou ascenseurs, etc.) } \\
\text { pour appuyer, adosser ou ranger les } \\
\text { lots selon leur statut. }\end{array}$ & $\begin{array}{l}\text { Éléments du bâti } \\
\text { (murs, poteaux, } \\
\text { ascenseurs, etc.) pour } \\
\text { appuyer, adosser ou } \\
\text { ranger les lots selon } \\
\text { leur statut. }\end{array}$ \\
\hline $\begin{array}{l}\text { Acheminement } \\
\text { des produits } \\
\text { traités vers les } \\
\text { véhicules par } \\
\text { destination } \\
\text { dans un temps } \\
\text { fort contraint. }\end{array}$ & $\begin{array}{l}\text { Gérer les fermetures en } \\
\text { faisant converger le } \\
\text { départ des flux des } \\
\text { produits traités. } \\
\text { Aiguiller les flux sur les } \\
\text { bons véhicules au } \\
\text { départ. }\end{array}$ & $\begin{array}{l}\text { Recours aux éléments du bâti créant un } \\
\text { goulet d'étranglement structurant un } \\
\text { point de passage unique pour s'assurer } \\
\text { de la convergence de la totalité des flux } \\
\text { et de leur controle. }\end{array}$ & $\begin{array}{l}\text { L'ascenseur. } \\
\text { Le quai des arrivées. } \\
\text { La cloison séparant } \\
\text { l'intérieur de } \\
\text { l'extérieur du quai. } \\
\text { Le système de jeu de } \\
\text { portes. }\end{array}$ \\
\hline
\end{tabular}

Dans cette approche, l'espace physique et organisationnel est considéré dans sa dimension de ressource à l'activité. À partir de cette déclinaison des liens rassemblant activité et éléments d'espace, des enseignements pour la conception sont possibles.

\subsubsection{Traduire les buts et les invariants opératoires en objectifs à satisfaire par la conception}

À partir des objectifs de travail que s'assignent les opérateurs dans leur action et des invariants opératoires, il est possible de dégager et formaliser des critères pour la conception. Ils sont à formuler du point de vue du concepteur. Leurs énoncés sont à construire autour d'objectifs ${ }^{9}$ à atteindre dans le travail de conception. Le recours à des verbes d'action est à envisager de façon à inviter directement le(s) concepteur(s) (ou les décideurs) à raisonner en termes d'aide et de ressource au service de l'activité des opérateurs.

Dans la démarche proposée, les buts des opérateurs doivent être traduits en objectifs à satisfaire, ou critères de conception, puis traduits en termes de premières propositions spatiales. Ce processus est illustré dans le tableau 2 pour le cas du tri postal. Les propositions spatiales sont des principes et des hypothèses d'aménagement à explorer. 
Tableau 2 : Identification des objectifs à satisfaire par la conception et des possibles réponses (ou traductions) spatiales.

Table 2: Identification of design targets and their possible spatial responses or translation

\begin{tabular}{|c|c|c|}
\hline $\begin{array}{l}\text { Rappels des buts } \text { (ou } \\
\text { objectifs de travail) des } \\
\text { opérateurs }\end{array}$ & 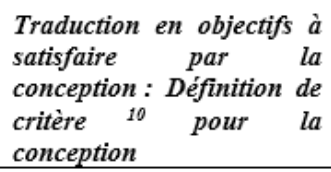 & $\begin{array}{l}\text { Premières propositions en termes de réponses } \\
\text { spatiales: principes et hypothèses } \\
\text { d'aménagement à explorer }\end{array}$ \\
\hline $\begin{array}{l}\text { Repérer, filtrer, contrôler et } \\
\text { hiérarchiser les lots de } \\
\text { courrier selon les } \\
\text { différentes priorités. }\end{array}$ & $\begin{array}{l}\text { Aider les agents à réaliser } \\
\text { l'activité de "l'aiguillage» } \\
\text { et la "priorisation» des } \\
\text { flux entrants. }\end{array}$ & $\begin{array}{l}\text { - Quai des arrivées. } \\
\text { - Cloison permettant de séparer et délimiter } \\
\text { dans l'espace les flux déposés par les véhicules } \\
\text { de ceux pris en charge par les agents. } \\
\text { - Système de jeu de portes. }\end{array}$ \\
\hline $\begin{array}{l}\text { Organiser visuellement la } \\
\text { progression de la prise en } \\
\text { charge des lots et faire } \\
\text { converger les flux des } \\
\text { produits traités en fin de } \\
\text { production. }\end{array}$ & $\begin{array}{l}\text { Aider les agents à gérer les } \\
\text { stocks dans le temps et } \\
\text { l'espace. }\end{array}$ & $\begin{array}{l}\text { - Espace présentant des éléments du bati } \\
\text { (murs, poteaux, ascenseurs, etc.) utilisés } \\
\text { comme appui pour les stocks pour les adosser } \\
\text { ou ranger les lots selon leur statut. }\end{array}$ \\
\hline $\begin{array}{l}\text { Acheminer les produits } \\
\text { traités vers les véhicules par } \\
\text { destination dans un temps } \\
\text { fort contraint. }\end{array}$ & $\begin{array}{l}\text { Aider les agents à gérer les } \\
\text { fermetures et faire } \\
\text { converger les flux sortants. } \\
\text { Aider les agents à gérer le } \\
\text { départ des flux. }\end{array}$ & $\begin{array}{l}\text { - Goulet d'étranglement spatial des flux (par } \\
\text { exemple un ascenseur ou autre élément de } \\
\text { convergence et de passage obligé des flux). } \\
\text { - Possibilité d'utilisation des deux quais } \\
\text { (arrivées et départs). } \\
\text { - Cloison séparant l'intérieur de l'extérieur du } \\
\text { quai. } \\
\text { - Système de jeu de portes. }\end{array}$ \\
\hline
\end{tabular}
espace (physique et organisationnel) adapté à l'activité. Elle est centrée sur la transposition des éléments de l'analyse (spatiale et de l'activité) dans un document à destination des concepteurs (et décideurs). La démarche consiste à mettre l'accent sur « les aspects non directement liés à la tâche, mais à l'obtention des conditions efficaces de sa réalisation " (Salembier \& Pavard, 2004, p. 92). L'objectif est d'orienter les concepteurs et les décideurs dans le travail de conception de l'artefact à partir de critères à satisfaire intégrant les conditions de réalisation de l'activité. Ils sont à intégrer au cahier des charges à l'attention des concepteurs.

Ces critères peuvent également servir dans un second temps, de support permettant d'initier un travail en groupe réunissant concepteurs, décideurs et opérateurs dans une réflexion pour le développement de l'activité. Cette partie est à outiller différemment. Elle concerne le travail de conception associant les différents acteurs concernés et les opérateurs et est développée ci-dessous.

\subsection{Viser le développement conjoint de l'activité et de l'espace de l'activité dans le travail de conception}

41 L'approche a consisté jusqu'à là à identifier et préserver ce qui fait ressource à l'activité (d'une façon intelligente). La démarche est maintenant à ouvrir sur ce qui peut être développé, modifié et innové dans et par la conception.

En effet, la conception ne doit pas seulement répondre aux besoins de l'activité, mais elle est à mettre dans une perspective développementale des activités. L'enjeu est de viser le « développement conjoint des situations et des activités 》 (Béguin, 2007, 2013; Béguin \& 
Cerf, 2004). La visée peut aussi être le " développement des individus et de leur situation " (Petit \& Dugué, 2013, p. 224). Nous pensons qu'il faut y rajouter une autre ouverture, celle du développement de l'espace de l'activité, en lien avec l'activité collective des sujets.

Cette considération conduit à engager une démarche qui associe tant les concepteurs que les opérateurs en articulant deux préoccupations :

La première implique les concepteurs et les opérateurs et consiste à instruire deux dimensions complémentaires :

- La dimension des affordances de l'espace, à penser au service de l'activité.

- La dimension des échanges et des communications médiatisés par l'espace.

La seconde vise à ouvrir un horizon pour le développement de l'activité et l'espace de l'activité au travers d'échanges et de discussions sur l'activité et l'espace de l'activité avec les opérateurs. L'objectif est d'engager avec les opérateurs un processus dynamique d'appropriation d'un espace à concevoir et susceptible de créer les conditions du développement de leur activité. L'enjeu est la construction des conditions pour le développement conjoint de l'activité et de l'espace de l'activité dans une approche émergente. L'espace de l'activité étant émergeant de l'activité.

Il s'agit de construire un dispositif d'intervention instaurant une dynamique de groupe de travail associant les opérateurs, les concepteurs et les décideurs autour de supports partagés pouvant prendre des formes différentes. Il peut s'agir de :

- documents de travail formalisant des éléments issus de l'analyse et en lien avec les pratiques d'espaces en situation de travail ;

- supports graphiques ou maquettes en vue de simulations de l'activité.

Sur un plan méthodologique, la démarche consiste dans un premier temps à construire un outillage constitué de supports de discussion élaborés à partir des éléments de l'analyse (spatiale et de l'activité). L'objectif est d'enclencher une discussion sur l'espace de l'activité à partir de l'exploitation des éléments de l'analyse. Le recours aux simulations est ensuite à mettre en œuvre avec l'objectif d'ouvrir sur le développement de l'activité et l'espace de l'activité. Les simulations sont alors à envisager comme une activité d'exploration des formes possibles de configuration de l'activité et de l'espace de l'activité. Un nombre significatif de réunions avec le groupe de travail est à envisager de façon à permettre une progression dans l'exploration des problèmes et des formes de réponses en termes d'activité et des espaces à penser pour l'activité. Le déroulé des séances du groupe de travail est à imbriquer avec des moments d'arbitrage et d'orientation stratégique à réaliser dans une instance de décision incluant la participation de l'ergonome.

\subsubsection{Exploiter et mettre les éléments d'analyse au service de la projection de l'espace de l'activité}

Il s'agit de mettre au service de la projection de l'espace de l'activité les éléments issus de l'analyse au travers de l'élaboration de supports à destination du groupe de travail. Les éléments de l'analyse formalisés à l'occasion de la construction du cahier des charges peuvent être partiellement repris pour enclencher la dynamique de discussion dans le groupe de travail réunissant opérateurs et concepteurs. Il est proposé d'instruire deux dimensions complémentaires, chacune apportant un éclairage spécifique sur l'espace de l'activité :

- La dimension des affordances de l'espace conçu 
- La dimension des échanges et des communications médiatisés par l'espace

Le premier axe consiste à explorer collectivement les dimensions d'affordance

Il s'agit d'explorer les qualités que l'espace à concevoir doit offrir. L'approche suppose l'engagement des concepteurs. Ils sont capables d'élaborer des propositions pour la conception d'espaces aidants au sens de Kirsh (1995). Pour concevoir des espaces (physiques et organisationnels) qui soutiennent l'activité, la présence des concepteurs et décideurs est nécessaire, mais elle ne suffit pas. La participation des opérateurs au sens de Daniellou (2007), reprenant Wilson (1991), est une condition pour explorer et décliner les propriétés d'affordance de l'espace conçu dans une optique de développement des schèmes d'utilisation. En effet, les opérateurs sont producteurs des schèmes d'utilisation dans la réalisation effective de leur activité. C'est bien par la mise à l'épreuve par l'activité que l'artefact peut évoluer vers «le statut d'instrument » (Clot, 2003).

L'espace à concevoir doit prendre en compte les objectifs ou les critères à satisfaire. La conception doit non seulement y répondre, mais aussi offrir des qualités et des affordances (Norman, 1993) pour le développement de l'activité. Pour aborder la qualité et les affordances que doit présenter l'espace conçu (Lefebvre, 1974), deux façons de procéder sont possibles: prendre en compte les formes existantes des réponses en matière d'espace et les reproduire, les explorer et ouvrir sur de nouvelles formes de traduction possibles.

L'analyse du travail permet de montrer au travers de la mise en lumière des buts et des invariants opératoires comment les liens sont tissés entre l'activité et un espace physique et organisationnel existant. L'exploration des possibilités nouvelles de retissage possible est à réaliser à partir d'une réflexion sur les affordances que l'espace conçu doit pouvoir offrir. Il s'agit de repérer ce qui fait sens dans le nouveau modèle d'espace et ce qui constitue une contrainte pour les acteurs. De ce point de vue, les formes d'affordance sont à interroger. Celles qui existent peuvent être reprises et valorisées. D'autres sont à créer ou modifier en fonction de l'évolution de l'activité et de ses exigences.

Le tableau 1 peut être ici à nouveau exploité et transposé en support pour ouvrir la discussion dans le groupe de travail. L'accent est à mettre sur les invariants opératoires et les artefacts spatiaux utilisés. Un exemple issu de l'intervention recherche réalisée dans le tri postal illustre le propos.

Les opérateurs ont repris dans le groupe de travail les objectifs de travail auxquels la conception doit répondre. Ils ont interrogé les schèmes d'utilisation actuels qu'ils ont retraduits différemment dans le modèle spatial du nouveau centre basé sur un centre de plain-pied. Les fonctions attribuées à l'espace physique ont été retrouvées, mais elles ont été déclinées autrement pour prendre en compte le choix immobilier du hall industriel (de plain-pied) en lieu et place du centre historiquement organisé sur deux niveaux. Par exemple, un goulet d'étranglement pour le passage des flux (assuré par l'ascenseur dans le centre ancien) a été retrouvé par la création d'un circuit qui rétrécit et présente un angle droit afin d'offrir une opportunité par l'espace aménagé d'organiser les lots avant et après le goulet. Un quai en profondeur ainsi qu'une paroi séparatrice comportant un nombre de portes précis et adapté aux volumes ont aussi été proposés. 
En mobilisant le groupe de travail sur les qualités requises pour le quai des arrivées, la réflexion menée dans le cadre de la conception du futur centre a conduit d'une part à retrouver des dimensions d'affordance existantes pour le quai, mais aussi à en proposer de nouvelles.

Parmi celles qui existent et à retrouver, il est possible d'évoquer le principe de séparation entre l'intérieur et l'extérieur du centre au travers d'une cloison délimitant clairement deux zones chacune spécifique à une activité. À l'extérieur, le déchargement des lots par les chauffeurs dans l'ordre d'arrivée des camions. À l'intérieur, l'activité contrôlée et organisée des lots par priorité réalisée par les agents. L'affordance offerte par l'artefact spatial permet ici l'articulation de deux activités intimement reliées, mais supposant des formes de gestion et de prise en charge des lots différenciées selon les acteurs. Elle offre une autonomie spatiale dans la gestion des priorités de chacun en permettant le passage des lots d'un acteur à l'autre en tenant compte de l'exigence temporelle de chacun.

Le groupe de travail a en même temps innové sur le statut et le rôle du quai arrivé et proposé une nouvelle affordance. Profitant de l'apport de l'analyse sur le double statut du quai des arrivées et sa transformation ponctuelle en quai pour les départs, les opérateurs ont ouvert la réflexion sur une autre proposition. Au-delà de sa fonction de quai dédié aux arrivées, ils ont envisagé la possibilité de lui conférer le rôle de plate-forme de transit pour gérer un flux particulier reçu par le centre sans pour autant nécessiter de traitement de tri. Il s'agit d'un flux courrier en développement en provenance de grandes entreprises qui ont recours à des logiciels (programmes de publipostage) permettant l'ordonnancement des lots par adresse. Les lots arrivent déjà triés par destination au centre en échange d'une ristourne importante sur la tarification de l'affranchissement. Ce flux transite par le centre. Il doit simplement être transféré du quai des arrivées vers le quai des départs sans aucune opération de traitement. Il requiert aujourd'hui une attention particulière et occupe les agents qui doivent assurer son transfert d'un bout à l'autre du centre tout en veillant à ne pas engorger l'intérieur du centre. L'idée d'un quai associé à une plate-forme de transit permettant de réceptionner et stocker ces lots le temps nécessaire avant leur départ sur les véhicules en partance a été explorée en simulation. Cela présente l'avantage d'éviter leur transfert d'un quai à l'autre par les agents. Ils gagnent ainsi du temps et évitent d'encombrer l'intérieur du centre d'un flux ne nécessitant pas de tri.

La démarche a visé la conception d'un espace physique et organisationnel présentant un « potentiel d'usages matériels ou imaginaires » (Lautier, 1999, p. 190). Des formes d'affordances ont été élaborées en vue d'utilisations retrouvées ou modifiées des différents éléments du bâti.

51 Cette manière d'explorer les affordances permet d'instruire les qualités de l'espace à concevoir. Autrement dit, de l'espace « donné » au sens de Lave (1988). 


\section{Le second axe consiste à explorer la dimension des échanges et des communications médiatisés par l'espace} communication pouvant passer par l'espace, les éléments issus de l'analyse spatiale de l'activité sont à mettre en discussion. Les échanges au travers des postures, les gestes, etc., en lien avec la dimension spatiale de l'activité constituent un matériau pouvant servir de support de discussion avec les opérateurs. La perspective est d'ouvrir la réflexion sur les impacts des modifications de l'espace sur l'activité et l'évolution de l'espace de l'activité. Pour la conception, cela se traduit alors par la prise en considération d'exigences de natures différentes. Ainsi, il est possible de mettre en lumière des exigences liées à la perception (relations visuelles, olfactives, sonores, etc.). Elles sont aussi des critères à prendre en compte par la conception.

Pour illustrer, le tableau 3 est construit à partir d'une typologie de critères propres à la communication médiatisée et permise par l'espace dans le cas du travail de tri. Ce tableau peut servir de repère pour débattre des formes de la communication non verbale.

Tableau 3 : Exploration des critères relatifs à la communication passant par l'espace. Table 3: Exploration of criteria relating to communication via space

\begin{tabular}{|l|l|l|}
\hline Exigences de visu & Critères pour la conception & $\begin{array}{l}\text { Réponses spatiales } \\
\text { possibles : principes d } \\
\text { hypothèses d'aménagement } \\
\text { à explorer }\end{array}$ \\
\hline $\begin{array}{l}\text { Sur les encours et les } \\
\text { stocks }\end{array}$ & $\begin{array}{l}\text { Permettre l'identification spatiale des } \\
\text { zones distinctes }\end{array}$ & $\begin{array}{l}\text { Délimitation par le bâti combiné à } \\
\text { système de signalétique } \\
\text { aérienne ou au sol }\end{array}$ \\
\hline $\begin{array}{l}\text { Sur les équipes des } \\
\text { chantiers stratégiques } \\
\text { d'homogénéisation }\end{array}$ & $\begin{array}{l}\text { Aider les agents à se rendre compte de dencement de la production au } \\
\text { travers de la communication verbale } \\
\text { (gestes visuellement intelligibles) }\end{array}$ & $\begin{array}{l}\text { Localisation centrale } \\
\text { Proximité } \\
\text { Transparence (paroi vitrée par } \\
\text { exemple) }\end{array}$ \\
\hline
\end{tabular}

\subsubsection{Construire un dispositif d'intermédiation par la simulation pour l'exploration des formes d'émergence de l'espace de l'activité}

Le recours aux techniques de la simulation est une pratique éprouvée en ergonomie ${ }^{10} \mathrm{La}$ simulation contribue à ce que Cerf, Prost, Barcellini, Barbier et Jeufroy (2015) qualifient d'activité d'intermédiation de l'ergonome qui vise la transformation des situations de travail. Elle contribue à impliquer les différents acteurs dans une organisation pour concevoir ensemble les conditions pour le développement des activités (Barcellini, Van Belleghem et Daniellou, 2013).

Rapportée à l'espace, la simulation permet un cadre pour explorer collectivement les possibles traductions spatiales des critères mis en avant précédemment. Accompagnés dans le cadre de la démarche d'intervention par l'ergonome et impliqués dans la structure projet, les opérateurs sont capables de mettre en débat les manières d'utiliser 
l'espace donné et d'anticiper les ajustements nécessaires dans une visée de transformation de l'activité. Il est ici proposé de l'envisager pour mettre en discussion l'activité et l'espace de l'activité tant sur le registre de sa dimension physique et organisationnelle que sur le registre immatériel des échanges et des communications verbales ou non dans le travail.

La démarche consiste à outiller la dynamique des groupes de travail au travers de supports et d'objets permettant la simulation de l'activité et de l'espace de l'activité. Par la simulation, un cadre d' « expérimentation ergonomique» (Maline, 1994) permet une forme de "mise en situation » (Béguin, 2007), condition pour ouvrir sur une dynamique d'échange (Maline, ibid.) portant sur l'espace de l'activité. En mettant en mots et en gestes l'activité des opérateurs dans sa dimension spatiale, la simulation ouvre sur une perspective d'activité collective de construction d'espaces de l'activité. Elle suppose (1) la construction de scénarios à simuler et (2) la formalisation de support(s) de simulation.

1. Les scénarios sont à construire en intégrant des repères connus par les opérateurs. Ils sont en lien avec la manière dont les opérateurs coordonnent leur action dans l'espace physique et organisationnel. Les scénarios à retenir doivent être issus du référentiel opératif commun (de Terssac \& Chabaud, 1990). Il s'agit de simuler des situations de travail connues et significatives dans le travail. Élaborés et retenus sur la base de «l'analyse préalable de situations de référence existantes» (Daniellou, 2007, p. 80 en référence à Daniellou, \& Garrigou (1993), les scénarios mettent en lumière les « sources de diversité et de variabilité " (Daniellou, 2004, p. 365). Des situations d'action caractéristiques (Daniellou, 2004) sont à simuler en évoquant le lien à l'espace matériel (physique et organisationnel).

2. Les supports de simulation sont des artefacts à construire. Ils peuvent prendre la forme de plans, de maquettes à différentes échelles ou de schémas fonctionnels. Ils sont à la fois un support d'échanges et d'action collective (Béguin, 2007) dans un groupe de travail. Leur fabrication est à réaliser de façon à intégrer des objets, des graphiques ou des supports connus et reconnus par les opérateurs. L'objectif est de permettre aux opérateurs qui les manipulent de retrouver des gestes réalisés dans l'activité. Autrement dit, ces supports doivent jouer un rôle "synchronisateur opératoire" (Darses \& Falzon, 1996) lors de la simulation des scénarios évoqués précédemment. Au travers de la manipulation de supports s'ancrant dans des gestes qui évoquent ceux réalisés au quotidien dans l'activité, un travail d'exploration de scénarios différents peut s'enclencher. Une manière de procéder consiste à rechercher et représenter des éléments de l'espace par de petits supports (en carton par exemple) aisément manipulables. Une autre façon peut être d'intégrer des outils habituels permettant de faire un lien avec l'espace et les transposer sur le support de simulation. Par exemple, des magnets utilisés pour représenter des activités ou des fonctions sur un tableau blanc dans une autre intervention portant sur la conception d'espaces de l'activité.

Pour illustrer ce propos, dans le cas de l'intervention menée dans les centres de tri du courrier, les objets à manipuler ont représenté :

- d'un côté, les différents chantiers de tri en intégrant l'évolution temporelle de leur traitement : chantiers en amont d'homogénéisation et de ventilation des lots, stocks et encours homogénéisés en amont de traitement, chantier de traitement automatique des plis de grand format, chantier de traitement automatique des plis de petit format, chantier manuel, chantier de ventilation en aval du chantier de traitement automatique des plis de grand format, chantier de ventilation en aval du chantier de traitement automatique des plis de petit format, etc. ; 
- de l'autre, les machines de tri et les véhicules. Les premières constituent des repères spatiaux qui s'imposent de par leur dimension (les machines de traitement des plis petits formats mesurent plus $42 \mathrm{~m}$ alors la machine de traitement des plis grand format avoisine les $100 \mathrm{~m}$ ). Elles donnent la mesure et l'échelle. Les véhicules apportent une indication sur la temporalité. Selon les tranches horaires, leurs arrivées ou départs du centre ainsi que le nombre et l'emplacement des véhicules sont différents. Leur place et leur nombre indiquent la tranche horaire et par conséquent le type de traitement pris en charge de façon prioritaire dans le centre.

Le support de simulation, confectionné à partir de plans, croquis ou maquettes d'étude, doit satisfaire trois exigences :

- Le choix de l'échelle doit être adapté au périmètre de la situation de travail à discuter ${ }^{11}$;

- Le support doit pouvoir faire l'objet de manipulation par les acteurs eux-mêmes. Il doit être un moyen de projection permettant une manipulation par chacun. Le support doit intégrer cette exigence ${ }^{12}$;

- Enfin, le support doit pouvoir être transportable ${ }^{13}$.

En manipulant les supports sur la base des scénarios, il est possible de mettre en mots et en gestes les modes de coordination et de communication verbale ou non médiatisée par l'espace (emplacement, exigence de visu, relation sonore, sensori-motrice, etc.). L'objectif consiste à engager un effort collectif de projection simultanée de l'activité et de l'espace sur la base de gestes de manipulation de supports représentatifs du quotidien de l'activité. En associant la manipulation des supports au récit sur l'activité, le support de simulation peut évoluer du statut d'artefact vers celui d'un outil permettant au groupe de travail d'explorer, d'instruire et délibérer sur les différentes possibilités d'émergence de l'espace de travail.

Accompagnés dans le cadre de la démarche d'intervention par l'ergonome et impliqués dans la structure projet, les opérateurs sont capables de mettre en débat les manières d'utiliser l'espace donné et d'anticiper les ajustements nécessaires dans une visée de transformation de l'activité. En contribuant à l'exploration de différentes hypothèses de transformation de l'espace en tant que dispositif physique et organisationnel, ils contribuent à concevoir des repères pour l'élaboration de nouveaux schèmes d'utilisation (Daniellou, 2007) dans le même temps qu'ils participent à la conception de l'artefact spatial. Il s'agit tout à la fois de convoquer des schèmes d'utilisation des artefacts spatiaux connus et d'en créer de nouveaux. Les échanges verbaux et non verbaux qui s'instaurent lors la simulation contribuent à évoquer ceux qui se créent dans l'espace de l'activité. Ce faisant, ils permettent d'engager une discussion qui contribue à penser simultanément l'évolution de l'activité et de l'espace de l'activité et contribuent à initier une dynamique pour une première forme de production spatiale locale telle que l'évoque Suchman (1996). C'est-à-dire ouvrir la réflexion autour d'un système artefactuel spatial donné puis modifié pour accueillir un espace émergent de l'activité. Cet espace émergent est alors un moyen pour construire et actualiser, dans le cours de l'action de simulation, « un référentiel contextuel partagé » (Salembier \& Pavard, 2004) dans la perspective de développement conjoint de l'activité et de l'espace.

Pour illustrer, reprenons le cas du tri postal.

Huit scénarios ont été explorés en groupe de travail (Figure 2) sur la base des différents critères élaborés en groupe de travail et ont permis d'arrêter une 
organisation de l'espace physique du centre (figure 3). Le support a été une maquette en carton au $1 / 100^{\mathrm{e}}$ permettant la manipulation de la configuration organisationnelle des différents chantiers du centre. Les chantiers et les machines ont été représentés sous forme de supports mobiles aisément manipulables par les opérateurs. Leur prise en main par les opérateurs reliant le geste et le récit du déroulé de l'activité au travers de la manipulation et de la transformation des chantiers dans le temps et l'espace a permis l'élaboration de scénarios et la discussion sur les avantages et les inconvénients de chacun des scénarios. Le groupe de travail s'est appuyé sur la simulation de l'activité collective dans le temps. La simulation a concerné les modifications des organisations des chantiers sur les différentes plages horaires sur les 24 heures. Chaque plage horaire correspond à un type de flux prioritaire et une organisation collective spécifique. C'est la discussion sur l'évolution temporelle de la forme et du nombre des chantiers représentés par de petits objets manipulables qui a été un moyen pour relier le geste de manipulation des chantiers sur maquette à un geste professionnel de construction du chantier dans l'activité collective. Cela a été le moyen de rassembler les opérateurs dans l'exploration des scénarios tout en ancrant leurs gestes de manipulation de la maquette dans un référentiel opératif commun des gestes réalisés dans leur quotidien du travail.

Figure 2 : Illustrations du travail de groupe sur support maquette en carton (cas du tri postal). Figure 2: Illustrations of working with a reduced model in the postal case study
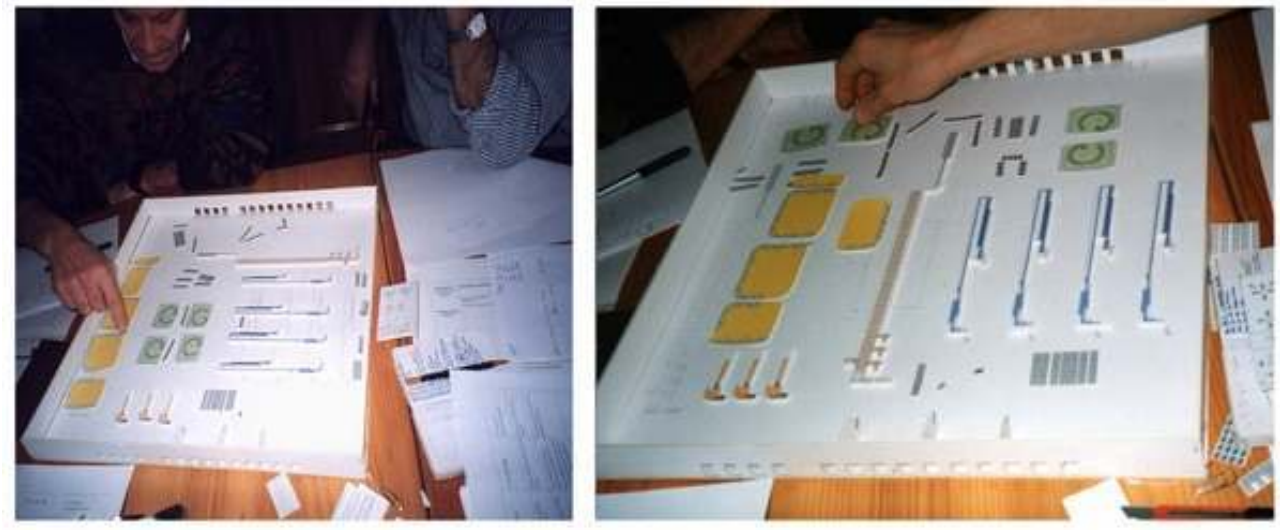

La simulation permet une première forme d'expérience de l'espace physique et organisationnel en phase de conception. Elle permet cette "mise en situation" mentionnée par Béguin (2007) dans une approche prospective (Maline, 1994) de l'espace de l'activité. En ce sens, elle permet des « simulations intérieures » (Davezies, 2014) par les opérateurs de leurs gestes et actions dans une perspective de développement de leur activité et de l'espace de leur activité. La mise en débat des modalités et des formes de « communication dans le travail» (Zarifian, 2010) peut alors s'opérer collectivement. Les verbalisations à l'occasion des simulations multi-supports permettent d'aborder les questions de communication et d'échanges non verbaux qui s'opèrent au travers de l'emplacement de chacun dans l'espace physique et organisationnel. De ce point de vue, le recours aux simulations multi-supports permet d'outiller le dispositif d'intervention en valorisant à la fois des propriétés d'émergence et de mise en situation (Salembier \& Pavard, 2004). 
Figure 3 : Illustration des résultats du travail de groupe : exemple de deux des huit scénarios élaborés en groupe de travail.

Figure 3: Illustrations of the results of the working group: two of the eight scenarios developed by the group

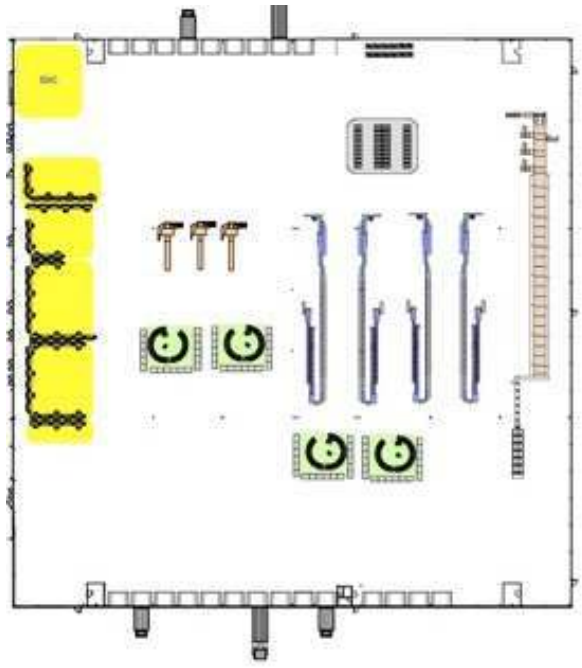

SCENARIO $n^{\circ} 4$

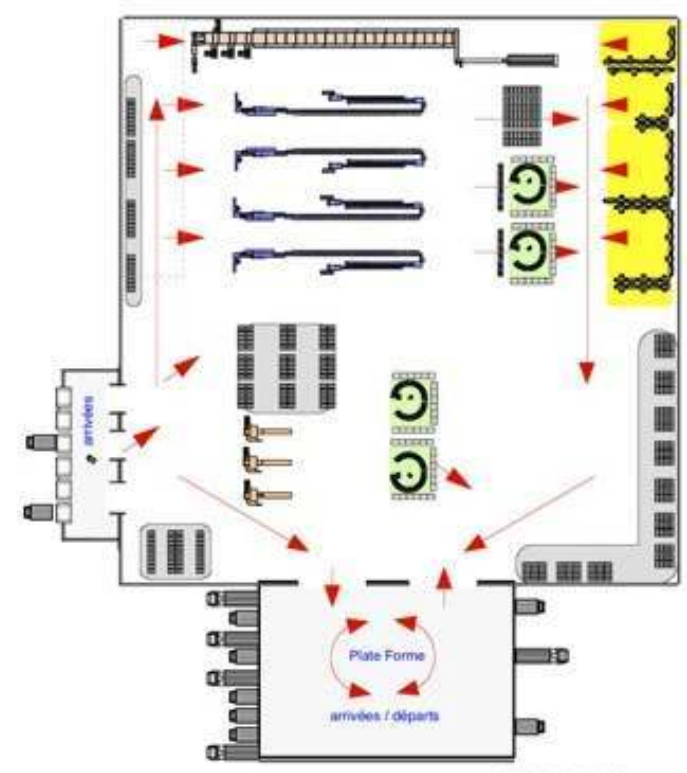

SCENARIO $n^{\circ} 8$

\section{engagés dans le groupe de travail. En confrontant les points de vue de chacun sur un support artefactuel (maquette ou autre support de simulation) jouant le rôle d'objet intermédiaire (Vinck, 2009), les scénarios d'exploration des nouvelles configurations de l'espace peuvent être instruits du point de vue de leur impact sur l'activité. L'artefact, utilisé pour la simulation, est un support d'échanges permettant leur focalisation (Béguin, 2007). «La simulation fonctionne comme un moyen pour "ouvrir l'espace de conception" ${ }^{14}$ et nourrir l'interaction (discussion et négociations) entre les acteurs engagés dans la conception autour de différentes alternatives possibles " (Salembier \& Pavard, 2004, p. 95). De ce point de vue, la simulation permet une coconstruction des compromis techniques, organisationnels et spatiaux entre des acteurs appartenant à des " communautés hétérogènes " (Béguin, 2007). Les leviers sont explorés simultanément sur les trois registres. Le choix est à opérer en recherchant le meilleur compromis possible pour l'ensemble des acteurs concernés par la démarche. En réunissant opérateurs, concepteurs et décideurs, la démarche consiste à construire et traiter ensemble un " problème de conception » (Béguin, 2014). L'impact sur l'activité n'est plus le seul problème des opérateurs. Il est partagé par le groupe de travail. De la même façon, l'impact sur le système spatial et technique n'est plus uniquement l'affaire des concepteurs ou des décideurs. Il est mis en discussion dans le groupe de travail.}

La reflexion est à ouvrir sur la multiplicité des angles d'approche des différents acteurs

\section{Discussion}

La discussion porte sur deux points. Le premier interroge l'approche instrumentale appliquée à l'espace notamment dans sa conception des systèmes d'instruments. Le second discute des apports et des limites de l'approche par l'espace de l'activité. 


\subsection{Apports} instrumentale et par l'enaction, vise à éviter le risque d'écarter ou de réduire la dimension spatiale à l'une de ses dimensions. Le recours à deux cadres distincts vise non pas de compléter l'un par l'autre, mais de mettre les deux approches en tension pour éclairer différemment la question spatiale et cela en déplaçant les questions d'un champ à l'autre. Chacun des cadres éclaire une facette de l'objet espace de l'activité dans le même temps qu'il met à l'ombre ses autres dimensions tant sur le plan de l'analyse de la situation de travail (Heddad, 2016) que de la conception.

La thèse défendue ici est que toute dynamique d'activité suppose une construction simultanée et imbriquée de l'espace et de l'activité. L'espace de l'activité comporte à la fois :

- une dimension artefactuelle composé de l'espace donné par l'organisation du travail, dont le bâti, et de l'espace modifié, ajusté et réorganisé par l'activité,

- une dimension émergente de l'activité au travers de l'espace agi et modelé par l'activité se formant dans les relations, coordination, coopération et toutes les formes de communications verbales ou non se créant dans l'activité.

L'approche instrumentale permet de saisir l'espace comme un artefact, matériel et organisationnel, modifié et assisté par et dans l'activité. Des schèmes d'utilisation selon la définition revisitée de Vergnaud (1996) peuvent lui être associés et éclairer le travail conception. 
71 La théorie de l'enaction de Varela et al. (1993) permet de saisir l'espace comme une configuration spatiale individuelle et collective émergente de l'activité. Dans cette approche, il y a un continuum entre le sujet percevant et les objets qui l'entourent dans l'ici et maintenant.

72 L'espace, un objet complexe, suppose une approche multidisciplinaire. Son exploration dans le champ de l'ergonomie sous un angle mettant en lumière sa complexité en lien avec la spécificité de l'activité est une première tentative de rapatrier et revisiter en ergonomie des notions propres à la complexité de l'objet « espace ».

\subsection{Limites}

73 L'activité réelle future ne se prévoit pas (Daniellou, 2007). En étant située, elle est relative à la situation réelle qui ne se prédit pas totalement. La focalisation en conception sur l'artefact conduit à considérer l'espace de l'activité au travers du prisme de la relation entre l'activité et l'artefact spatial. Or, l'espace de l'activité n'est pas seulement un espace physique et organisationnel, mais il émerge des relations, interactions, coordinations et communication non verbale mises en œuvre en situation réelle. Travailler est «une activité dirigée en situation réelle» (Clot, 1999, p. 94-95). "Le réel, c'est l'action de réalisation - jamais prévisible - qui met le sujet aux prises avec les réalités objectives du monde des choses et des hommes, occasions et obstacles à son développement. » (Ibid., p. 94).

74 Sur le registre spatial, tout ne peut être anticipé. L'espace de l'activité est une production locale au sens de Suchman (1996) dans un effet de structure globale rassemblant les opérateurs et leur espace tout au long de la réalisation de l'activité. Même si l'espace est pensé en amont dans une visée de prise en compte des schèmes et de leur développement, l'espace de l'activité sera (toujours en partie) une production issue de la réalisation de l'activité en situation. D'un côté, l'action du sujet en situation ne répond pas à un plan prédéfini (Cerf \& Meynard, 2006; Suchman, 1987). Elle n'est pas programmable. De l'autre, l'espace de l'activité est le produit de l'activité. En tant qu'expérience du sujet au monde dans la réalisation de son travail, il est émergent à l'activité au sens de la théorie de l'enaction (Varela et al., 1993). Cette part non anticipable conduit à une prudence méthodologique. Intégrer les opérateurs dans le processus de conception non seulement dans la conception du nouveau système artefactuel, mais en les amenant à interroger et repenser l'activité est incontournable. Acteurs dans la conception et de l'activité, les opérateurs sont les garants d'une continuité entre la conception et la situation de travail. La simulation est un moyen efficace pour articuler la phase de la conception avec l'expérience spatiale même si elle ne se substitue pas totalement à l'activité des opérateurs en situation réelle.

Une autre limite concerne l'effort de catégorisation opérée dans la démarche d'intervention. Comme toute nouvelle catégorisation, certaines délimitations ou choix de catégories peuvent être questionnés. Ainsi, l'organisation a été traitée d'un point de vue instrumental, c'est-à-dire sous le double angle : celui de l'organisation inscrite dans toute forme matérielle spatiale par l'organisation du travail et les intentions des concepteurs et décideurs, mais aussi celui de l'organisation réelle mise en œuvre dans l'opérationnalité du déroulé de l'activité par les opérateurs qui la modifient. Ils l'ajustent et transforment les affectations fonctionnelles de «l'espace donné » par l'organisation. Le parti pris a été de traiter l'organisation comme rattachée à l'artefact spatial. L'organisation aurait pu 
aussi être considérée comme émergente de l'activité et dans ce cas, regardée sous la théorie proposée par l'enaction. Le choix fait dans ce texte tente de garder une certaine cohérence avec l'idée qu'il faut tenir compte des invariants, mais pas nécessairement de tout ce qui peut émerger en situation, même si l'objectif est de viser un espace favorable à ces émergences. C'est cette visée d'un espace favorable à des émergences qui pourrait rendre intéressant le choix de regarder l'organisation sous l'angle de l'enaction.

\section{Perspective} ergonomie et souvent malmenée en architecture, mérite une valorisation. En défendant un point de vue spécifique sur le développement conjoint de l'activité, au travers du concept d'espace de l'activité, l'objectif est de contribuer à enrichir tant l'analyse du travail que la conduite de projet lors de l'intervention en ergonomie.

démarche consiste à analyser une situation existante et outiller un dispositif d'intervention dans le cadre de la conception pour contribuer au développement de l'activité et l'espace de l'activité. Qu'en est-il lorsque la conception concerne une activité à imaginer sans possibilité d'appui sur un existant? Comment construire la démarche face à la difficulté des opérateurs à penser des espaces pour une activité cible à créer et pour laquelle ils ne disposent pas de référentiel en termes de pratique spatiale ? C'est le cas des activités délicates qui ne se produisent que de façon rare et exceptionnelle comme la gestion des crises où la situation de travail n'existe pas encore, mais qu'il faut toutefois anticiper pour penser l'espace de l'activité. De ce point de vue, l'impossibilité de recourir à l'analyse de l'activité et spatiale d'un dispositif physique et organisationnel, ressource à l'activité, suppose d'innover sur le plan méthodologique pour imaginer l'activité et l'espace de l'activité.

Dans la posture qui est la nôtre, le rôle de l'ergonome est celui d'un initiateur facilitateur d'un processus de développement des capacités de penser et concevoir conjointement les transformations de l'activité et les pratiques d'espaces dans une visée développementale. Une piste peut être esquissée. Nous pensons que la démarche est à centrer sur la recherche d'une construction ad hoc de supports de simulation multiples avec l'objectif de permettre aux opérateurs un ancrage dans leur expérience de leur espace de l'activité actuelle pour engager une dynamique de réflexion et de projection d'un espace de l'activité à imaginer. Se profile ici l'ambition d'un nouvel article à formaliser.

Un autre axe à développer concerne la question des apprentissages, des règles de métier et des savoirs partagés qui se forment à partir de la réflexion sur l'espace de l'activité et sa mise en œuvre. Ce registre n'a pas été traité. Il peut contribuer à apporter un autre éclairage notamment sur la manière de construire l'intervention pour concevoir l'espace de l'activité. La simulation est par exemple source d'apprentissage pour penser l'espace de l'activité. Elle est en effet à la fois une activité productive contribuant au travail de conception, mais elle est aussi à visée constructive (Samurçay \& Rabardel, 2004) contribuant à l'augmentation des savoirs et de l'expérience des membres du groupe de travail que ce soit les opérateurs ou les concepteurs sur la manière dont l'espace de l'activité se crée, se forme et évolue en lien avec l'activité. En contribuant à construire un cadre pour penser le développement « des ressources de leurs actions » (Béguin, 2007), la démarche permet aux opérateurs, mais aussi aux concepteurs et décideurs engagés dans 
la démarche le « développement des conceptualisations et des compétences » (Pastré, 1999, mentionné par Béguin 2007). Cette voie reste à explorer.

\section{BIBLIOGRAPHIE}

Amalberti, R. (1996). La conduite des systèmes à risques. Paris : PUF, Coll. Le travail Humain.

Arnoud, J. \& Falzon, P. (2013). Changement organisationnel et reconception de l'organisation : des ressources aux capabilités. Activités, 10(2), 109-130. http://activites.revues.org/760 Barcellini, F., Van Belleghem, L., \& Daniellou, F. (2013). Les projets de conception comme opportunité de développement des activités. In P. Falzon (Ed.), Ergonomie Constructive (pp. 191-206). Paris : PUF.

Béguin, P. (2006). In search of a unit of analysis for designing instruments. Artefact, 1(1), 32-38.

Béguin, P. (2007). Innovation et cadre sociocognitif des interactions concepteurs-opérateurs : une approche développementale. Le Travail Humain, 4(70), 369-390.

Béguin, P. (2013). La conception des instruments comme processus dialogique d'apprentissage mutuels. In P. Falzon (Ed.), Ergonomie constructive (pp. 147-160). Paris : PUF.

Béguin, P. (2014). Learning during design through simulation. In O. Broberg, N. Fallentin, P. Hasle, P.L. Jensen, A. Kabel, M.E. Larsen \& T. Weller (Eds.), Proceedings of Human Factors in Organizational Design and Management, XI and Nordic Ergonomics Society Annual Conference, 46 (pp. 867-872).

Béguin, P., \& Cerf, M. (2004). Formes et enjeux de l'analyse de l'activité pour la conception des systèmes de travail. Activités, 1(1), 54-71. http://activites.revues.org/1156.

Béguin, P., \& Weill-Fassina, A. (1997). De la simulation des situations de travail à la situation de simulation. In P. Béguin, \& Weill-Fassina (Eds.), La simulation en ergonomie : connaître, agir et interagir (pp. 5-28). Toulouse : Octarès.

Bourmaud, G. (2013). De l'analyse des usages à la conception des artefacts : le développement des instruments. In P. Falzon (Ed.), Ergonomie constructive (pp. 161-173). Paris : PUF.

Cerf, M., \& Meynard, J.M. (2006). Les outils de pilotage des cultures : diversité de leurs usages et enseignements pour leur conception. Natures Sciences Société, 14, 19-29.

Cerf, M., Prost, L., Barcellini, F., Barbier, M., \& Jeufroy, M-H. (2015). Scaling user's participation : design-use relations in transition towards the ecologization of agriculture. Participating in Innovation, innovating in participation. IEA 13 conference. Melbourne, Australia.

Chalandon, X. (2007). Conscience de la situation : invariants internes et invariants externes. Contribution de l'ergonomie cognitive et de l'ingénierie à la conception de systèmes d'aide à la gestion des environnements dynamiques. Thèse de doctorat en ergonomie, Cnam.

Clot, Y. (1999). La fonction psychologique du travail. Paris : PUF. 
Clot, Y. (2003). La catachrèse entre réel et réalisé. Contribution d'un psychologue du travail. In Y. Clot \& R. Gori (Eds.), Catachrèse : éloge du détournement (pp. 11-26). Nancy : Presses Universitaires de Nancy.

Clot, Y., \& Gori, R. (Eds.) (2003). Catachrèse : éloge du détournement. Nancy : Presses Universitaires de Nancy, coll. Langage-Cognition-Interaction.

Daniellou, F. (1988). Ergonomie et démarche de conception dans les industries de process continus, quelques étapes clefs. Le travail Humain, 51(2), 184-194.

Daniellou, F. (2004). L'ergonomie dans la conduite de projets de conception de systèmes de travail. In P. Falzon (Ed.), Ergonomie (pp. 359-373). Paris : PUF.

Daniellou, F. (2007). Des fonctions de la simulation des situations de travail en ergonomie. Activités, 4 (2), 77-83, http://activites.revues.org/1696.

Daniellou, F., \& Garrigou, A. (1993). La mise en œuvre des situations passées et des situations futures dans la participation des opérateurs à la conception. In A. Weill-Fassina, P. Rabardel, \& D. Dubois (Eds.), Représentation pour l'action (pp. 359-374). Toulouse : Octarès.

Darses, F., \& Falzon, P. (1996). La conception collective : une approche de l'ergonomie cognitive. In G. de Terssac \& E. Friedberg (Eds.), Coopération et Conception (pp. 123-135). Toulouse : Octarès.

Davezies, P. (2014). Travail et émancipation. Conférence Université Paris 1. Centre Mahler, Paris. Dejean, P.H., Pretto, J., \& Renouard, J.P. (1988). Organiser et concevoir les espaces de travail. Montrouge : Anact.

Endsley, M. (1995). Toward a Theory of Situation Awareness. Human Factors, 37(1), 65-84.

Falzon, P. (2013). Pour une ergonomie constructive. In P. Falzon (Ed.), Ergonomie constructive (pp. 1-15). Paris : PUF.

Folcher, V., \& Rabardel, P. (2004). Hommes-Artefacts-Activités : Perspectives instrumentales. In P. Falzon (Ed.), L'ergonomie (pp. 251-268). Paris : PUF.

Garrigou, A. (1992). Les apports des confrontations d'orientation socio-cognitives au sein de processus de conception participatifs : le rôle de l'ergonomie. Thèse de doctorat en ergonomie, Cnam.

Goffman, E. (1974). Les rites d'interaction. Paris : Éditions de Minuit.

Hall, E. T. (1966/1971). La dimension cachée. Paris : Points seuil.

Heddad, N. (2016). L'espace de l'activité, de l'analyse à la conception. Thèse de doctorat en ergonomie, CRTD Cnam.

Heddad, N. (soumis). L'espace de l'activité : Une construction conjointe de l'activité et de l'espace. Kirsh, D. (1995). The intelligent use of space. Artificial Intelligence, 773, 31-68.

Lautier, F. (1999). Ergotopiques. Sur les espaces des lieux de travail. Toulouse : Octarès.

Lautier, F. (2000). La situation française : manifestations et éclipses de la figure du Maître d'Ouvrage. In M. Bonnet \& F. Lautier (Eds.), Les maîtrises d'ouvrage en Europe, évolutions et tendances (pp. 9-18). Paris : PUCA.

Lautier, F. (2001). Penser l('espace d)e travail. In F. Hubault (Ed.), Comprendre que travailler c'est penser, un enjeu industriel de l'intervention ergonomique (pp. 109-119). Toulouse : Octarès.

Lave, J. (1988). Cognition in Practice. Mind, Mathematics and Culture in Everyday Life. Cambridge : Cambridge University 
Lefebvre, H. (1974, 2000). La production de l'espace. Paris : Anthropos.

Lefort, B. (1982). L'emploi des outils au cours de tâches d'entretien et la loi de Zipf-Mandelbrot. Le Travail Humain, 45(2), 307-316.

Maline, J. (1994). Simuler le travail. Lyon : ANACT.

Martin, C. (2000). Maîtrise d'ouvrage, maîtrise d'œuvre : construire un vrai dialogue. La contribution de l'ergonome à la conduite de projet architectural. Toulouse : Octarès.

Norman, D. A. (1993). Things that make us smart. Reading, MA : Addison-Wesley.

Pastré, P. (1999). La conceptualisation dans l'action : bilan et nouvelles perspectives. In P. Pastré (Ed.), Apprendre des situations. Éducation permanente $n^{\circ} 139,13-36$.

Petit, J., \& Dugué, B. (2013). Structurer l'organisation pour développer le pouvoir d'agir : le rôle possible de l'intervention en ergonomie. Activités, 10(2), 210-228, http://activites.revues.org/816.

Piaget. J., (1936). La naissance de l'intelligence chez l'enfant. Lausanne : Delachaux et Niestlé.

Quéré, L. (1997). La situation toujours négligée ? Réseaux, 15(85), 163-192.

Rabardel, P. (1995). Les hommes et les technologies, approche cognitive des instruments contemporains. Paris : Armand Colin.

Salembier, P., \& Pavard, B. (2004). Analyse et modélisation des activités coopératives situées. Evolutions d'un questionnement et apports à la conception. Activités, 1(1). http:// activites.revues.org/1170.

Salembier, P., \& Zouinar, M. (2004). Intelligibilité mutuelle et contexte partagé, Inspirations conceptuelles et réductions technologiques. Activités, 1(2), 64-85. http://activites.revues.org/1243

Samurçay, R., \& Rabardel, P. (2004). Modèles pour l'analyse de l'activité et des compétences : propositions. In R. Samurçay \& P. Pastré (Eds.), Recherche en didactique professionnelle (pp. 163-180). Toulouse : Octarès.

Suchman, L. (1987). Plans and Situated Actions : The Problem of Human Machine Interaction. Cambridge : Cambridge University Press.

Suchman, L. (1996). Constituting shared workspaces. In Y. Engeström \& D. Middleton (Eds.), Cognition and communication at work (pp. 35-60). New-York : Cambridge University Press.

Terssac (de), G., \& Chabaud, C. (1990). Référentiel opératif commun et fiabilité. In J. Leplat \& G. De Terssac (Eds.), Les facteurs humains de la fiabilité dans les systèmes complexes (pp. 110-139). Toulouse : Octarès.

Van Belleghem, L. (2012). Simulation organisationnelle : innovation ergonomique pour innovation sociale. In M-F. Dessaigne, V. Pueyo, \& P. Béguin (Eds.), Innovation et Travail : Sens et valeurs du changement. Actes du $46^{\text {ème }}$ Congrès de la Self. Lyon.

Varela, F., Thompson, E., \& Rosch, E. (1993). L'inscription corporelle de l'esprit. Sciences cognitives et expérience humaine. Paris : Seuil.

Vergnaud, G. (1990). La théorie des champs conceptuels. Recherches en didactique des mathématiques, 10(2-3), 133-170.

Vergnaud, G. (1996). Au fond de l'action, la conceptualisation. In J.-M. Barbier (Ed.), Savoirs théoriques, savoirs d'action (pp. 275-292). Paris : PUF. 
Vergnaud, G. (2013). Pourquoi la théorie des champs conceptuels ? Infancia y Aprendizaje, 36(2), 131-161. DOI : 10.1174/021037013806196283.

Vinck, D. (2009). De l'objet intermédiaire à l'objet frontière : vers la prise en compte du travail d'équipement. Revue d'anthropologie des connaissances, 3(1), 51-72.

Wilson, J. (1991). Participation : A framework and a foundation for ergonomics? Journal of Occupational Psychology, 64, 67-80.

Zarifian, P. (2010). La communication dans le travail. Communication et organisation, $38 \mid 2010$, 135-146.

\section{NOTES}

1. Les projets immobiliers sont en effet menés en France avec la spécificité d'une forte séparation des responsabilités et des tâches entre les acteurs représentant d'un côté les propriétaires de l'édifice, la maitrise d'ouvrage et de l'autre, les acteurs en charge de sa conception, la maîtrise d'œuvre (Lautier, 2000). Maitres d'ouvrage et d'œuvre sont tous deux des acteurs institués une fois que la décision et le projet sont enclenchés en vue de construire un bâtiment (Ibid.).

2. Martin (2000) montre comment une intervention ergonomique peut s'inscrire dans un projet architectural dans le cadre de la commande publique, c'est-à-dire des marchés publics encadrés par la loi sur la maitrise d'ouvrage publique (MOP) en France. Il s'agit bien souvent d'importants projets de construction de bâtiments destinés à devenir des lieux dédiés au travail.

3. La notion de «schème " est introduite par Piaget (1936) dans son étude chez les enfants. Pour Vergnaud (1990), les schèmes sont organisateurs de l'activité. La notion de schème est reprise par Rabardel (1995) pour analyser les activités avec instrument. Vergnaud définit le schème comme une « organisation invariante de l'activité pour une classe de situations donnée » (2013, p. 138).

4. Pour Endsley (1995), la conscience de la situation s'opère au travers de la perception de l'environnement (espace et temps) permettant la compréhension de la situation et l'anticipation de ses états futurs.

5. Goffman (1974) défend l'idée qu'un lieu par son organisation et l'environnement qu'il offre peut contribuer à amplifier l'action. Les lieux de rencontre de hasard, de flânerie, les banques, etc. jouent un rôle dans la manière dont se réalise l'action. Il montre que le lieu participe des interactions des hommes à un moment donné.

6. La «proxémie ", est « l'ensemble des observations et des théories de l'usage de l'espace » (Hall 1966/1971, p. 129). Elle montre l'importance de la communication qui passe par les corps ou les distances entre les personnes. Elle relie l'ensemble à la culture. Nous l'adoptons ici dans le cadre des organisations de travail où ces éléments répondent aussi à des logiques propres à l'activité et l'organisation du travail.

7. Pour repérer les invariants organisationnels, le recours à des sites de référence est utile. Disposer d'un échantillon d'un à trois sites choisis sur le critère de leur représentativité par rapport au modèle d'espace et d'organisation du travail constitue une façon de faire. L'objectif est de disposer des moyens permettant de repérer ce qui est propre à l'activité et cela indépendamment du système organisationnel spatial local. Dans le cas de l'étude sur les centres de tri, un échantillon de 6 sites a permis de cibler au final 3 centres pour lesquels des analyses détaillées ont été élaborées. Les sites ont été choisis sur la base de 3 critères (type de bâti, densité urbaine, performance (qualité et productivité)).

8. Le tableau 1 ne reprend ni la règle d'action ni les règles d'ajustement (ou possibilités d'inférences). La première se concentre autour de l'obligation de gérer en continu les priorités des lots courrier selon leur état de prise en charge dans le traitement. Elle est un dénominateur 
commun à toutes les étapes de tri. De la même façon, les possibilités d'inférences concernent les ajustements opérés en situation tout le long de la réalisation de l'activité sans apporter une modification spécifique du dispositif spatial.

9. L'enjeu est d'aider le concepteur à réaliser un lien entre les réponses spatiales qu'il peut proposer en termes de fonctionnalités ou de qualification spatiales et l'activité des opérateurs. L'objectif est de l'inviter à s'interroger sur les impacts des choix qu'il opère sur l'activité des opérateurs.

10. De nombreux travaux en ergonomie montrent l'intérêt de passer par la simulation en conception (Garrigou, 1992 ; Maline, 1994 ; Béguin \& Weill-Fassina, 1997 ; Daniellou, 2007 ; Van Belleghem, 2012 ; Barcellini, Van Belleghem, \& Daniellou, 2013).

11. À l'échelle des flux ou de l'organisation d'un service, un $1 / 200^{\mathrm{e}}$ ou un $1 / 100^{\mathrm{e}}$ constitue une échelle pertinente pour explorer les liens entre l'activité et les questions d'espaces. Si la réflexion vise des relations entre équipes dans un même service, un support de simulation au $1 / 50^{\mathrm{e}}$ ou $1 / 25$ e est plus approprié. Si la discussion porte sur la disposition des outils au niveau des postes, un support confectionné dans une échelle variant entre $1 / 10^{\mathrm{e}}, 1 / 5^{\mathrm{e}}$, voire à l'échelle $1 / 1$ est à envisager. Dans un groupe de travail avec des opérateurs, passer d'une échelle à l'autre peut s'envisager. Cela suppose toutefois une organisation au préalable de façon à disposer des supports appropriés à temps.

12. Sa taille est à définir de façon à permettre une utilisation dans un groupe de travail composé généralement de 6 à 12 personnes. Cette condition contribue à préciser et arrêter une échelle adaptée à la fois à la situation à simuler et à la situation de simulation.

13. Bien souvent, le support est à confectionner en dehors de l'entreprise. Se pose alors la question de son transport. Un support conçu en kit ou démontable peut être une façon d'opérer pour réduire la contrainte du transport.

14. Le mot "espace" est utilisé ici par les auteurs dans un autre registre. Il semble faire référence aux marges de manœuvre et opportunités possibles en conception.

\section{RÉSUMÉS}

La dimension spatiale est propre à toute situation d'activité. Elle est reconnue comme partie intégrante de la situation de travail même si le concept d'espace est peu clarifié en ergonomie. Ce texte propose une démarche pour une conception conjointe de l'activité et l'espace au travers de la notion de l'espace de l'activité. Le concept s'inscrit dans un double cadre théorique et méthodologique a) l'approche instrumentale est exploitée pour saisir la dimension matérielle et organisationnelle de l'espace considéré comme un artefact auquel peuvent être associés des schèmes d'utilisation $b$ ) le recours à la théorie de l'enaction vise à saisir la dimension émergente de l'espace autour des relations qui se construisent dans le faire de l'activité et qui constituent une forme de communication non verbale. L'idée défendue est que toute activité se réalise dans, et détermine, un espace matériel et relationnel au travers des actions, des échanges entre les sujets ou entre les sujets et les objets qui les entourent. Ce texte propose une démarche pour intervenir en conception avec l'objectif d'articuler le déroulé de l'activité et la dynamique spatiale de l'activité. En conséquence, il s'agit de concevoir d'une part, l'espace, en tant que dispositif matériel et organisationnel, comme un support à l'activité dans une approche instrumentale et développementale des artefacts et des activités. D'autre part, il s'agit de prendre en compte la dimension émergente de l'espace de l'activité. L'approche proposée invite à penser 
conjointement l'espace et l'activité dans une visée de développement de l'activité et de l'espace de l'activité.

Spatial dimension is the proper of any working situation. The space of any activity requires consideration of the activity itself in relation to its surrounding space. This article proposes a concept of space of activity that is embedded in a dual theoretical and methodological framework. The instrumental approach captures the physical and organizational dimension of space. Enaction theory captures the emerging dimension of activity space as different forms of relation and communication. In this paper we discuss the approach of intervening in design, with the objective of focusing on the flow and spatial dynamics of the activity. We put forward the idea that any activity takes place in, and determines, a material and immaterial space through actions, relationships between subjects or between subjects and surrounding objects. It is therefore necessary on the one hand to design the space as a material and organizational device, as support for the activity in an instrumental and developmental approach to activity and, on the other hand, to take into account the emerging dimension of the activity's space. The proposed approach calls for a joint consideration of space and activity with a view to developing the activity and its spatial dimension.

INDEX

Mots-clés : espace de l'activité, espace physique et organisationnel, espace émergent, conception instrumentale, enaction, développement conjoint

Keywords : the space of the activity, physical and organizational space, emerging space, instrumental design, enaction, joint development

\section{AUTEUR}

NADIA HEDDAD

Cnam-crtd / Université Paris 1 FCPS 